Revue des patrimoines

$36 \mid 2018$

Les archives photographiques de presse, pratiques comparées et enjeux méthodologiques

\title{
Les archives photographiques de presse, pratiques comparées et enjeux méthodologiques
}

Éditorial

Press Photo Archives, Practices Compared and Methodological Questions

Isabelle-Cécile Le Mée

\section{OpenEdition}

Journals

\section{Édition électronique}

URL : http://journals.openedition.org/insitu/17487

DOI : $10.4000 /$ insitu. 17487

ISSN : $1630-7305$

\section{Éditeur}

Ministère de la Culture

\section{Référence électronique}

Isabelle-Cécile Le Mée, « Les archives photographiques de presse, pratiques comparées et enjeux méthodologiques », In Situ [En ligne], 36 | 2018, mis en ligne le 15 octobre 2018, consulté le 24 septembre 2020. URL : http://journals.openedition.org/insitu/17487 ; DOI : https://doi.org/10.4000/ insitu. 17487

Ce document a été généré automatiquement le 24 septembre 2020.

\section{(c)}

In Situ Revues des patrimoines est mis à disposition selon les termes de la licence Creative Commons Attribution - Pas d'Utilisation Commerciale - Pas de Modification 4.0 International. 


\section{Les archives photographiques de presse, pratiques comparées et enjeux méthodologiques}

Éditorial

Press Photo Archives, Practices Compared and Methodological Questions

Isabelle-Cécile Le Mée

Nous remercions le comité éditorial d'In Situ. Revue des patrimoines d'avoir choisi de consacrer un numéro à un domaine jusqu'alors absent de ses colonnes, la photographie, et plus particulièrement, un de ses usages, la photographie de presse.

(fig. 1) 


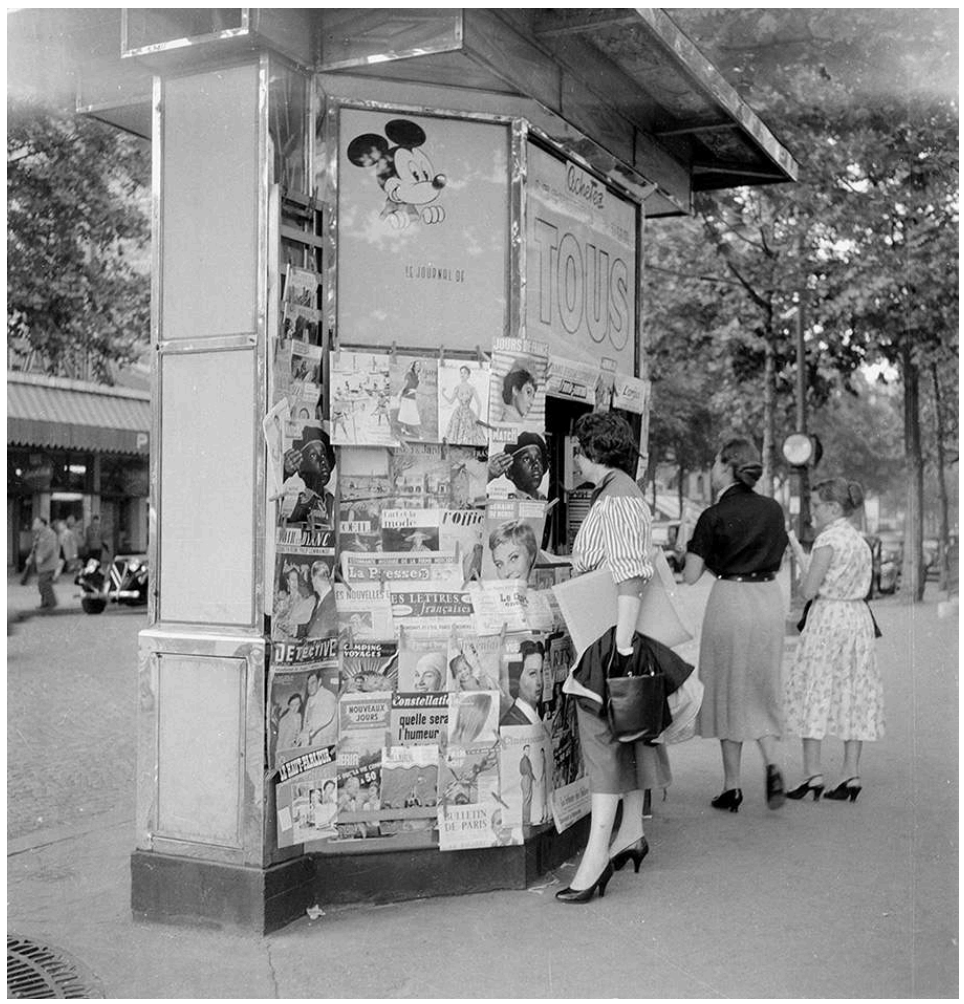

André Papillon, kiosque à journaux, tirage argentique.

(c) Succession André Papillon, musée Nicéphore Niépce, ville de Chalon-sur-Saône.

Intitulé Les Archives photographiques de presse, pratiques comparées et enjeux méthodologiques, ce numéro s'inscrit dans le prolongement de journées d'études organisées conjointement par la Mission de la photographie du ministère de la Culture et la Mission de la photographie des Archives nationales, les 24 et 25 novembre 2015. Ces journées se proposaient de faire émerger les grandes questions que pose la gestion de ces fonds argentiques, de réfléchir à des réponses adaptées aux problématiques soulevées et éclairées par la confrontation des expériences. Surtout, nous voulions aborder ces questions avec les producteurs des fonds, les représentants d'agences, des photographes, des responsables de services photographiques de journaux.

Plus généralement, nous souhaitions valider l'idée de la mise en œuvre d'une politique plus globale de collecte et de dépôt d'archives de presse en lien avec les producteurs, réfléchir à la rédaction d'un vade-mecum spécifique à l'accueil et au traitement de ces fonds, proposer des outils facilitant leur traitement et leur valorisation.

\section{L'avenir contrarié des archives photographiques de presse}

Depuis près d'un siècle, la presse est une grande consommatrice de photographies. C'est donc tout naturellement que les journaux, revues et groupes de presse ont constitué d'importants stocks de photographies. Beaucoup de titres se sont dotés de services photographiques fonctionnant avec des photographes salariés tout en récupérant des photos auprès d'agences ou de photographes indépendants. Ainsi 
mêlées, les photographies étaient classées selon la nature des besoins, souvent par thématiques, par noms de personnes ou de lieux.

Avec le temps, de disparitions de titres en rachats et regroupements, de nombreuses archives peinent à être localisées quand elles n'ont pas disparu, emportant avec elles un pan de notre histoire collective, de notre mémoire commune. Certaines en revanche sont encore détenues précieusement par des journaux et groupes de presse toujours actifs comme Paris Match, qui exploite ses archives, L'Équipe, qui conserve par ailleurs les plaques ayant servi à la publication du quotidien Excelsior.

Soucieux d'assurer une pérennité à leurs archives argentiques, les entreprises de presse, comme les auteurs, se tournent vers des établissements publics ou privés pour les vendre, les donner ou simplement les déposer. C'est souvent au moment d'un déménagement que la question de leur devenir se pose. Par ailleurs, depuis plusieurs années, l'approvisionnement en photographies se fait en numérique, rendant obsolète et lourd le fonctionnement de photothèques argentiques (fig. $2,3,4)$.

Figure 2

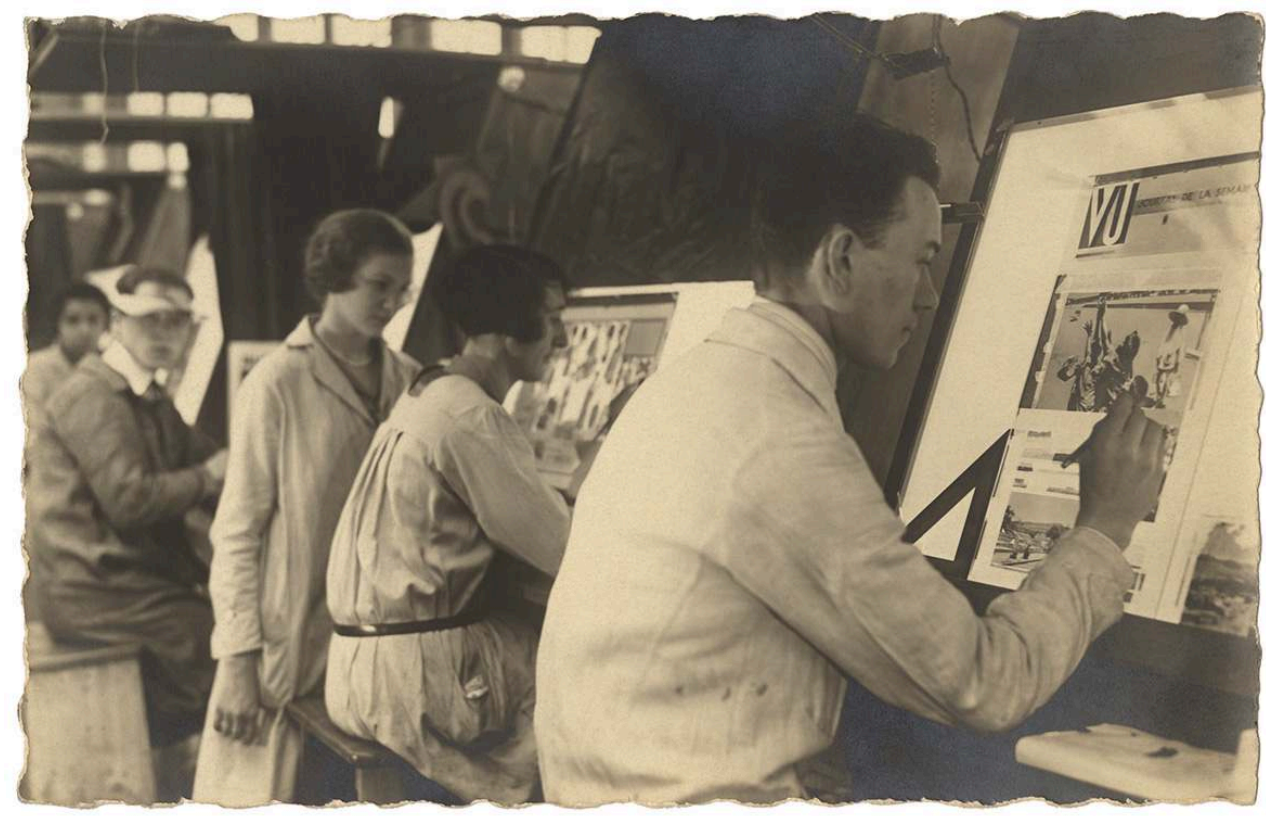

Roger Lemaire, fabrication du magazine Vu, années 1930, carte postale photographique, collection Musée Nicéphore Niépce.

(c) Musée Nicéphore Niépce, Ville de Chalon-sur-Saône. 
Figure 3

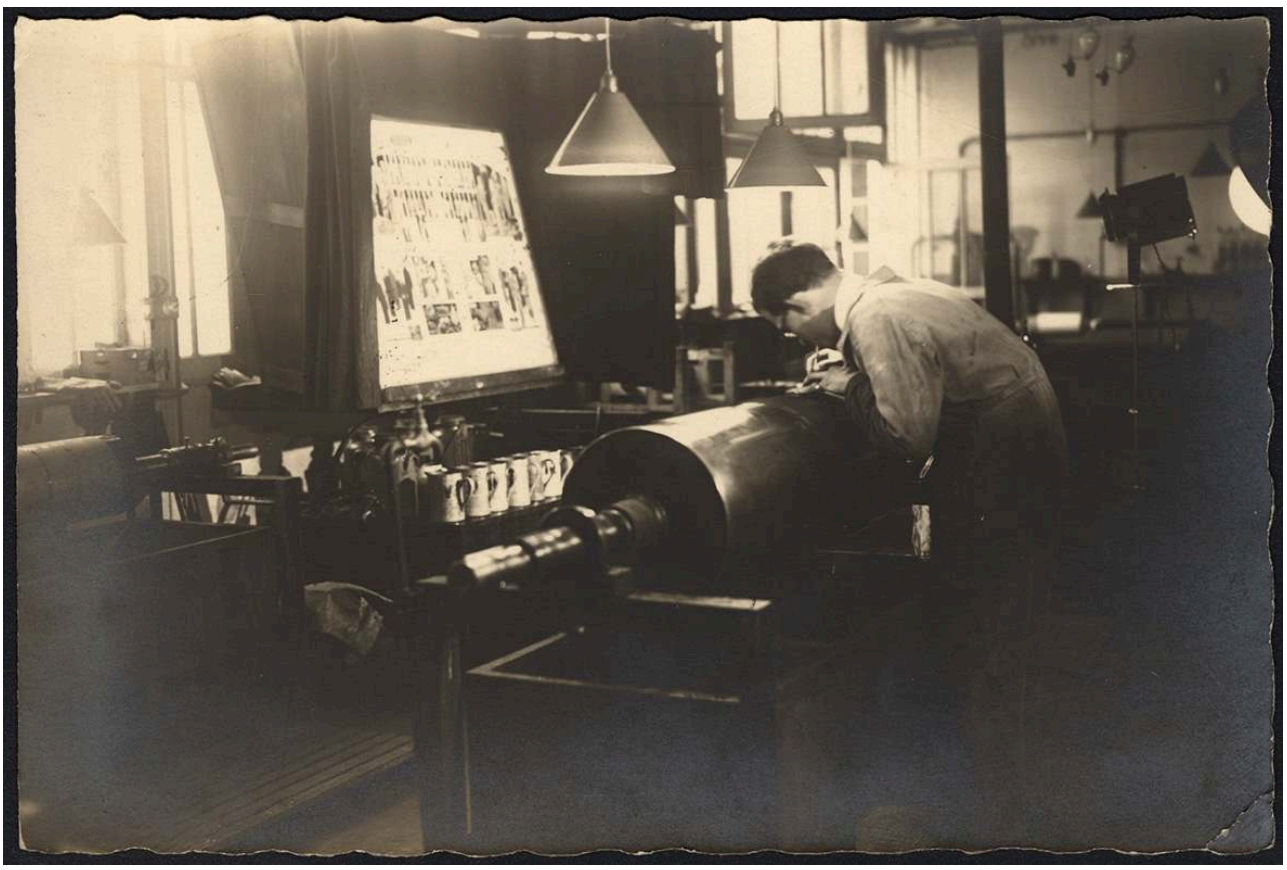

Roger Lemaire, fabrication du magazine $V u$, années 1930, carte postale photographique, collection Musée Nicéphore Niépce.

(c) Musée Nicéphore Niépce, Ville de Chalon-sur-Saône.

Figure 4

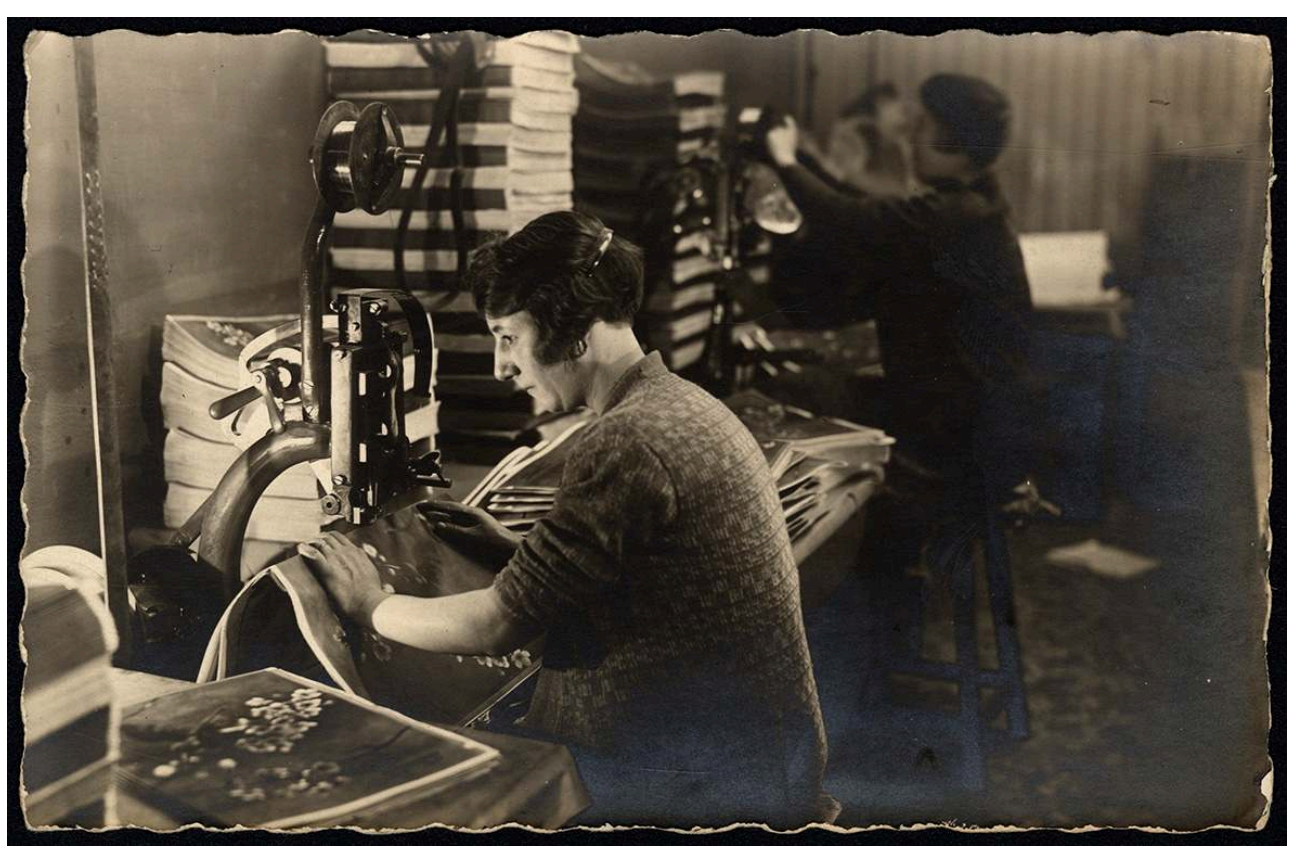

Roger Lemaire, fabrication du magazine Vu, années 1930, carte postale photographique, collection Musée Nicéphore Niépce.

(c) Musée Nicéphore Niépce, Ville de Chalon-sur-Saône.

Une première enquête réalisée conjointement par la Mission de la photographie du ministère de la Culture et la mission de la Photographie des Archives nationales, au printemps 2015, auprès des services d'archives départementales a permis de constater 
qu'une quarantaine d'entre eux sur les soixante-six qui ont répondu conservaient un ou plusieurs fonds entrant dans cette catégorie ${ }^{1}$. À titre d'exemple, les archives photographiques de Ouest-France (édition de la Manche) sont conservées par les archives départementales de la Manche, celles de Sud-Ouest (édition des Pyrénées atlantiques) par les archives départementales des Pyrénées-Atlantiques, les archives photographiques de La Dépêche du Midi ont été déposées, selon l'édition, aux archives départementales de la Corrèze, de la Haute-Garonne, du Lot. La même logique de dépôt est constatée pour La Montagne et ses éditions locales. Quelques photothèques de titres nationaux ont aussi été versées dans divers services. Citons celles de L'Humanité, déposées aux archives départementales de Seine-Saint-Denis, du Petit Parisien et du Parisien libéré aux Archives nationales. Le Jardin des modes est conservé par l'Institut des mémoires de l'édition contemporaine (IMEC, abbaye d'Ardenne). La Bibliothèque historique de la Ville de Paris abrite six millions de clichés du fonds France-Soir, la Bibliothèque nationale de France conserve quant à elle les fonds photographiques de L'Aurore ou du Journal....

Souvent volumineuses et hétérogènes, mal authentifiées, les archives de presse peinent à trouver preneur, et quand elles sont collectées, leur conservation et leur traitement obligent à la mise en œuvre de processus lourds et coûteux adaptés à leur spécificité. Le monde des archives, des musées ou des bibliothèques n'est pas naturellement familier du monde de la presse et les premiers sont souvent peu au fait des modalités de production ou d'exploitation des seconds. La poursuite de la collecte de ces fonds passe pourtant par l'appréhension des modalités de leur production, qui sont, dans le cas des archives photographiques de presse, très complexes car relevant conjointement de plusieurs acteurs (photographes, agences, journaux...).

Quelques premiers retours d'expérience nous avaient permis d'entrevoir les difficultés auxquelles se heurtaient les responsables de ces fonds. Deux écueils majeurs concernaient le difficile traitement scientifique et documentaire d'ensembles souvent volumineux et hétéroclites et la méconnaissance de l'environnement juridique attaché aux images, ce qui entravait toute tentative de valorisation.

Les centres d'archives récupèrent "des masses", parfois des ensembles organisés, souvent des lots de photographies, parfois anonymes, en vrac, sans plan de classement d'origine ni inventaire, et hélas! généralement dépourvues d'informations qui permettraient de considérer correctement leur environnement juridique.

Les quatorze articles réunis dans ce numéro s'inscrivent dans trois grands axes de réflexion retenus par l'appel à contributions : cinq articles abordent l'organisation de la collecte, la conservation, le traitement intellectuel des fonds, quatre articles s'intéressent aux problèmes juridiques attachés à la conservation des objets et aux droits afférents aux ensembles ou aux images. Enfin, cinq contributions abordent la valorisation possible des photographies de presse par le biais de mises en ligne sur des sites dédiés, d'expositions, ou de créations de structures adaptées.

\section{Collecte, conservation, traitement intellectuel des fonds}

Les contributeurs évoquent leur première confrontation à ce type d'archives volumineuses et hétéroclites, regroupant souvent des items de différentes natures (négatifs sur différents supports, tirages, diapositives, tirages isolés ou reportages 
complets) répondant à une pluralité d'usages. Comment, dès lors, les trier et les organiser? Comment hiérarchiser? Prioriser le traitement? Comment conserver et traiter chaque item en préservant la cohérence d'acquisition ou de constitution des ensembles? Quelle logique de traitement? Quels outils adapter ou concevoir? Pour les fonds provenant de photothèques de journaux, il faut aussi prendre en compte la complexité de l'environnement juridique des images. L'absence d'informations relatives à la propriété des photographies ou aux droits d'auteur associés aux images pouvant en interdire toute valorisation éventuelle, cette question mérite une attention particulière.

La prise en main des archives photos du quotidien L'Humanité, riche de plus de deux millions d'images reçues en dépôt aux archives départementales de Seine-Saint-Denis en 2003 concentre toutes ces interrogations. Elle a contraint Joël Clesse et Maxime Courban, qui en ont été chargés, à adapter les méthodes de travail. Ils reviennent aussi dans leur article sur le défi consistant à assurer la maitrise matérielle et intellectuelle d'une masse de documents dépourvue d'inventaire tout en maintenant la communicabilité et l'utilisation quotidienne des images. Pour Agathe Demersseman, au musée de la Résistance nationale (Champigny-sur-Marne), il a aussi fallu faire face à un fonds d'ampleur, celui dit du Matin, riche de 320000 négatifs, entré par dépôt du journal L'Humanité en 1987 (don en 1997). Hétérogène, arrivé en vrac sans inventaire, avec des photographies provenant de différents producteurs, cet ensemble s'est révélé complexe à traiter. Son article présente avec force détails la réflexion entreprise par le musée pour dégager les enjeux d'une telle prise en main dans le cadre du projet scientifique et culturel (PSC) de l'établissement, déterminer les priorités de traitement et de valorisation comme les moyens à mettre en œuvre pour y répondre.

Avec le texte de Sandrine Bula l'approche est plus historique. Elle dresse un panorama des fonds photographiques de presse conservés aux Archives nationales provenant du Petit Parisien, du Parisien libéré, du Journal ou de fonds d'agences et revient sur l'entrée de ces ensembles aux Archives nationales dans le cadre de la politique de sauvegarde des fonds de presse impulsée dans les années 1950 ou par le biais de versements effectués par la Société nationale des entreprises de presse (SNEP) chargée dès 1946 de la gestion des biens de presse confisqués à la Libération dont elle évoque l'histoire et les objectifs.

Si les trois premiers articles traitent de photothèques de journaux, Angelina Meslem, Wilfrid Eon et Clémentine Vialar présentent le fonds d'un photographe de presse, Jean Lattès (1917-1996), dont les archives ont été données en 2011 aux archives départementales des Yvelines. Recevoir plus de 200000 clichés a imposé aux archivistes, là encore, d'engager une réflexion nouvelle sur leur pratique. Ils ont décidé de la mener avec une spécialiste de la photographie habituée au traitement de la photographie au sein de collections muséographiques. Aux côtés des préoccupations de conservation, se sont posées des questions relatives au traitement intellectuel d'un ensemble photographique cohérent mais protéiforme. La compréhension du fonds repose sur une maîtrise de l'environnement et des modalités de production comme des besoins et des enjeux de diffusion, des usages et destinations des photographies produites. Ainsi, l'article revient sur la nécessité d'accompagner la collecte d'un travail d'enquête ce qui n'a pu être mené pour le fonds Lattès et qui a eu pour conséquence d'altérer la compréhension immédiate des pièces entre elles ainsi que le classement initial conçu pour la gestion du fonds et la diffusion. Dans le cas des archives de presse, 
la connaissance des contextes de production s'avère essentielle surtout quand celle-ci est plurielle (travail pour une agence, en indépendant, pour des entreprises, personnel...).

Cette première partie se clôt par l'article de la chercheuse Monica Di Barbora qui dresse un état des lieux de la recherche dans ce domaine en Italie, et évoque la difficile collecte de ces archives en mettant l'accent sur le destin des photothèques du quotidien l'Unità et de ses éditions locales.

\section{Nature juridique des fonds/Détermination des droits et exploitation des fonds}

Les aspects juridiques mobilisent les responsables de fonds photographiques et suscitent les inquiétudes. La gestion juridique des archives photographiques est indissociable de leur conservation et de leur valorisation. Dans le cas des archives de presse, ces questions prennent une ampleur nouvelle. L'authentification des producteurs, des auteurs, la connaissance ou non de la généalogie des archives, de leur transmission de titre en titre sont autant de freins potentiels à leur exploitation.

Ces questions touchent à la propriété des supports matériels conservés et aux droits d'auteur afférents. La première relève du Code civil, les seconds du Code de la propriété intellectuelle.

Si le photographe est le plus souvent propriétaire des items dont il fait don ou qu'il dépose, la question est moins tranchée en ce qui concerne les photothèques de journaux. Celles-ci, comme nous l'avons déjà évoqué, sont constituées d'images de provenances diverses produites dans des contextes d'agences différents et nombre de clichés, dans le cadre d'accords avec les agences fournisseuses et les photographes, auraient dû être restitués.

Par conséquent, une photothèque de journal peut-elle légitimement conserver les tirages qu'elle a reçus dans le cadre de son activité initiale, ou encore, le dépôt ou le don d'un tel ensemble à un centre d'archives est-il légitime?

Comme le précise l'article de Samuel Bonnaud Leroux, la diversité des acteurs du secteur du photojournalisme ainsi que la diversité des pratiques des agences, non homogènes et pas référencées, rendent difficile la réponse à ces questions.

Dès lors, comment ne pas imaginer que la difficulté à définir un environnement clair et borné ne soit pas un frein à la collecte de tels fonds? La crainte de revendications est réelle. La récupération d'archives de journaux a donné lieu par le passé à des passes d'armes entre photographes, agences et journaux (Ex: Herald Tribune). L'obligation de régulariser, la peur du démantèlement sont réelles, conduisant certains à ne pas médiatiser ni communiquer tout ou partie des ensembles qu'ils conservent. Fait regrettable tant ces photothèques, comme objets constitués, apportent de précieux témoignages sur l'évolution des pratiques journalistiques et éclairent sur l'évolution des regards portés sur l'information.

David Pouchard rappelle les grands principes qui régissent le droit d'auteur appliqué à la photographie. Être propriétaire d'un support (négatif, tirage...) n'implique pas que l'on détienne les droits d'auteur attachés. Il rappelle donc la nécessité pour les détenteurs de fonds d'obtenir les autorisations d'exploitation nécessaires avant tout usage. Dans le cas des archives de presse, cette question est particulièrement épineuse 
surtout lorsqu'aucun élément contractuel, ou aucun lien avec les producteurs des fonds, n'existe. Comment procéder alors? Démanteler et rendre ce qui ne peut être valorisé ? Rechercher les auteurs et les ayants droit concernés puis procéder à une régularisation pièce à pièce?

(fig. 5)

Figure 5

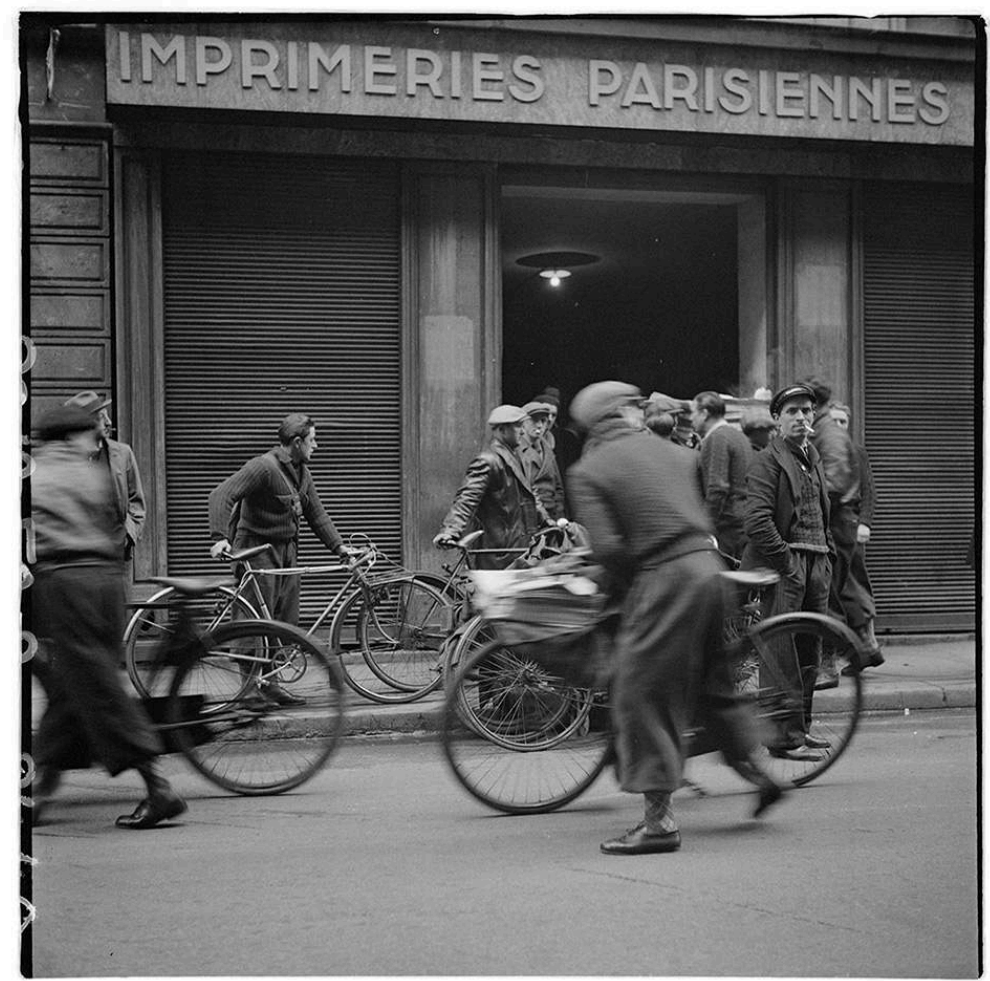

André Papillon, Livreurs de journaux devant les imprimeries parisiennes, tirage argentique. (c) Succession André Papillon, musée Nicéphore Niépce, ville de Chalon-sur-Saône.

Nous souhaitions compléter cette approche en nous tournant vers les producteurs de ces fonds, les représentants d'agences et les auteurs dont des tirages se trouvent aujourd'hui conservés dans de telles archives.

Et que pensent de ces situations les représentants des agences ou les ayants droit de photographes? Delphine Desveaux dresse un historique de l'agence Roger Viollet avant d'évoquer l'évolution de sa pratique commerciale qui voulait notamment que les tirages prêtés aux utilisateurs soient restitués après utilisation. Même manière de faire chez Rapho où les photographes étaient propriétaires des tirages qu'ils confiaient à l'agence pour en assurer la diffusion et en réclamer la restitution. Francine Deroudille, fille de Robert Doisneau et ancienne rédactrice chez Rapho, évoque le lien fort qui unissait Doisneau à son agence avant de revenir sur ces pratiques commerciales et donner sa position d'ayant droit. Comment faire, donc, avec des tirages non restitués et intégrés à des fonds presse conservés aujourd'hui dans des centres d'archives ou des musées? Delphine Desveaux comme Francine Déroudille optent pour des régulations sous forme de conventions de dépôt (fig. 6). 
Figure 6

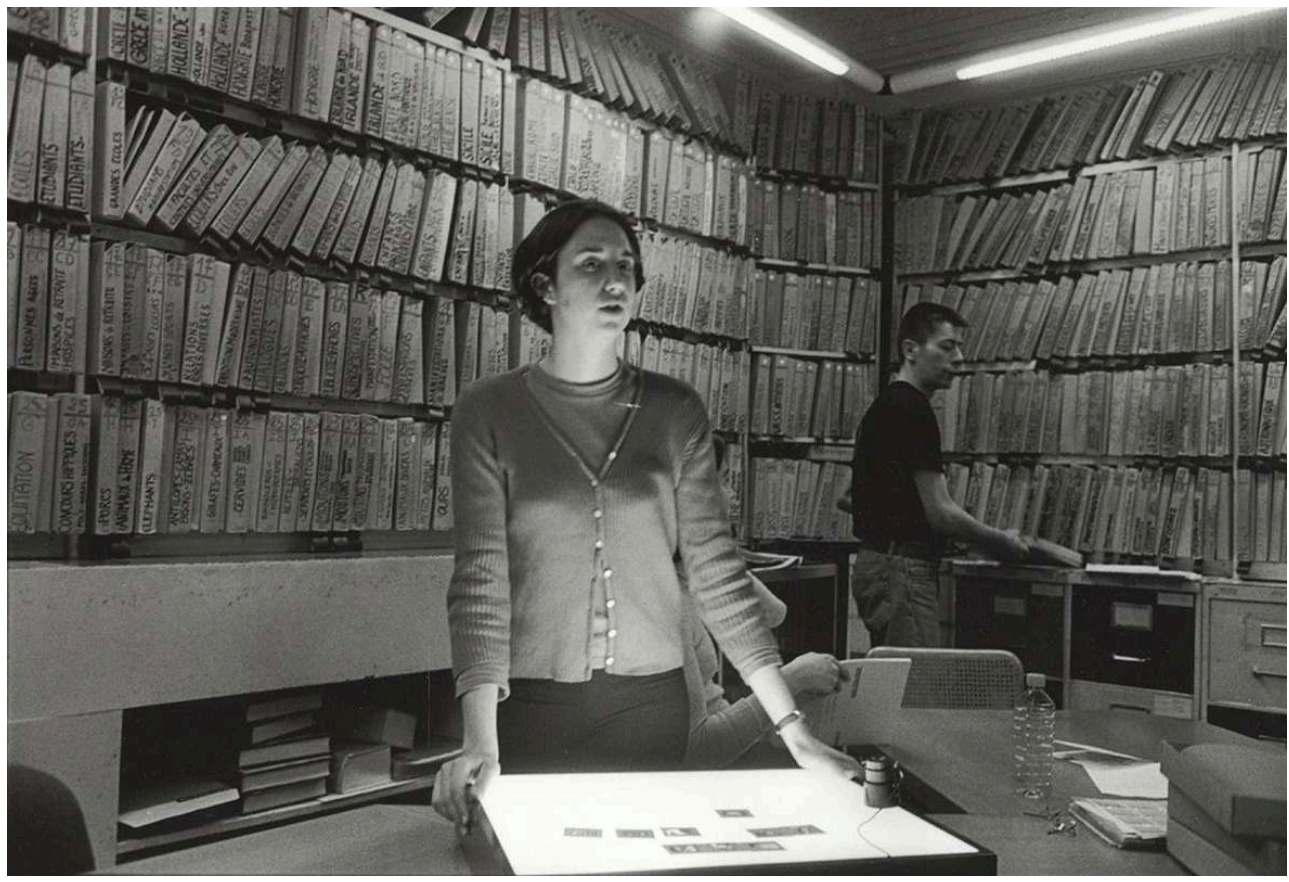

Dans les locaux de l'agence Rapho, années 1970.

Phot. François Le Diascorn. (c) François Le Diascorn.

La pluralité des fonctionnements administratifs et commerciaux des agences empêche toute généralisation et rend plus difficile encore la tâche des responsables de fonds amenés à distinguer les provenances de clichés afin d'en individualiser le traitement. Cette difficulté majeure nourrit le désir de voir élaborer un outil recensant les agences et leurs pratiques, précisant les liens contractuels unissant les photographes aux agences ou listant les modalités de cession des photographies dans le cadre de l'activité commerciale. Gageure ou utopie? Nous sommes, hélas! conscients de la difficulté à mettre en œuvre ce type d'outil pourtant nécessaire tant la vie des agences et des journaux est faite de rachats, de fusions ou de disparitions...

\section{Valoriser les archives photographiques de presse}

Une fois traités, quelle valorisation envisager pour ces fonds, pour leurs auteurs, pour cet usage de la photographie? Comment doit être conçue leur numérisation? Quelle présentation imaginer? Sur quels supports? Comment valoriser plus spécifiquement les photographies dans ces ensembles? Comment relier les items à l'œuvre des différents auteurs? Comment exposer des fonds presse?

Sylvain Besson rappelle la place importante donnée à la photographie de presse au musée Nicéphore Niépce (Chalon-sur-Saône), d'abord à travers les collections qui comptent quelques corpus de tirages de presse, mais aussi les revues photographiques ou les archives de photographes indépendants travaillant pour la presse; dans l'activité de valorisation, ensuite, par des expositions conçues comme des expériences d'immersion complexe, de découverte et d'exploration de la démarche d'auteur ou de revues par exemple mais aussi par le biais de dispositifs multimédias innovants intégrés aux parcours des expositions ou visibles et consultables en ligne, de manière à 
rendre compte de l'histoire des revues, de leur contexte de production, des spécificités de leur maquette et de leur réception par les lecteurs de l'époque (fig. 7, fig. 8).

\section{Figure 7}
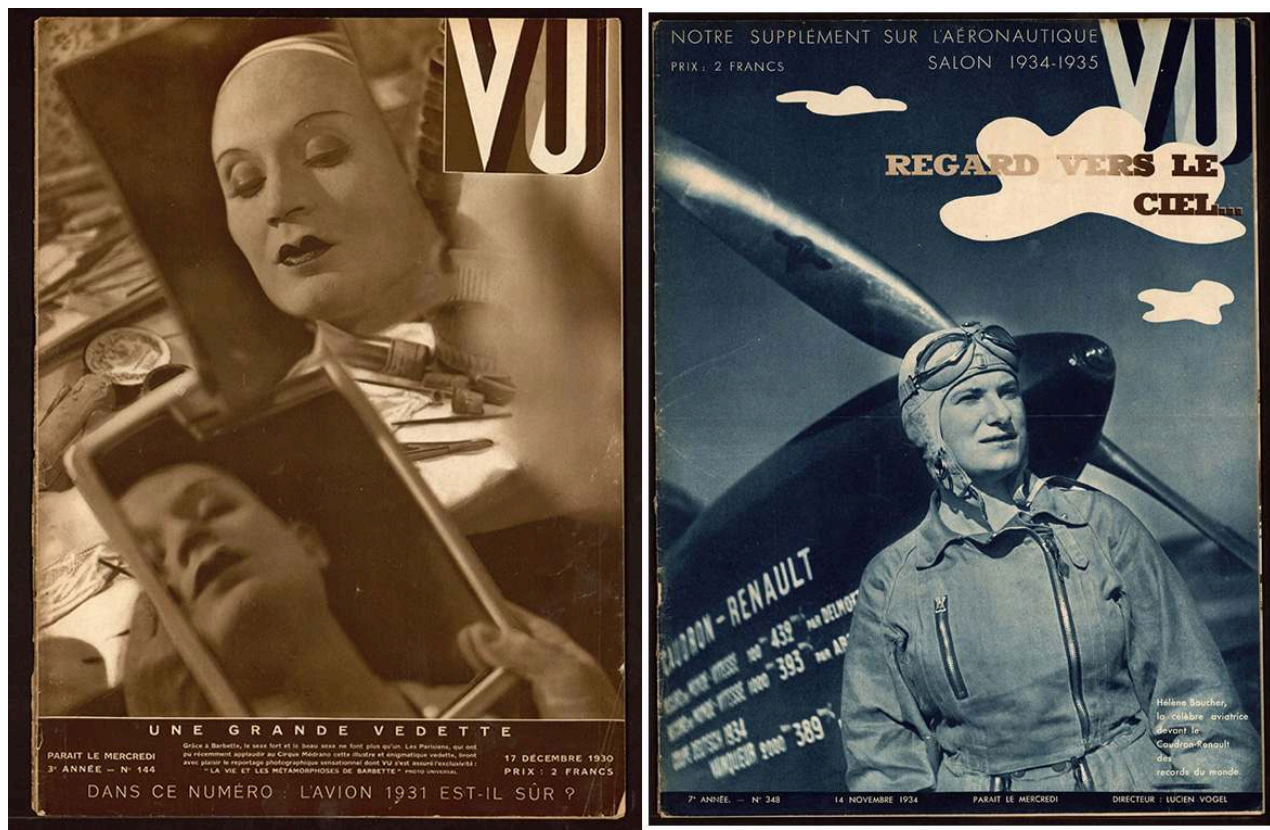

À gauche, couverture du numéro 144 du magazine $V u, 17$ décembre 1930. À droite, couverture du numéro 348 du magazine $V u, 14$ novembre 1934

(c) Musée Nicéphore Niépce, ville de Chalon-sur-Saône.

Figure 8
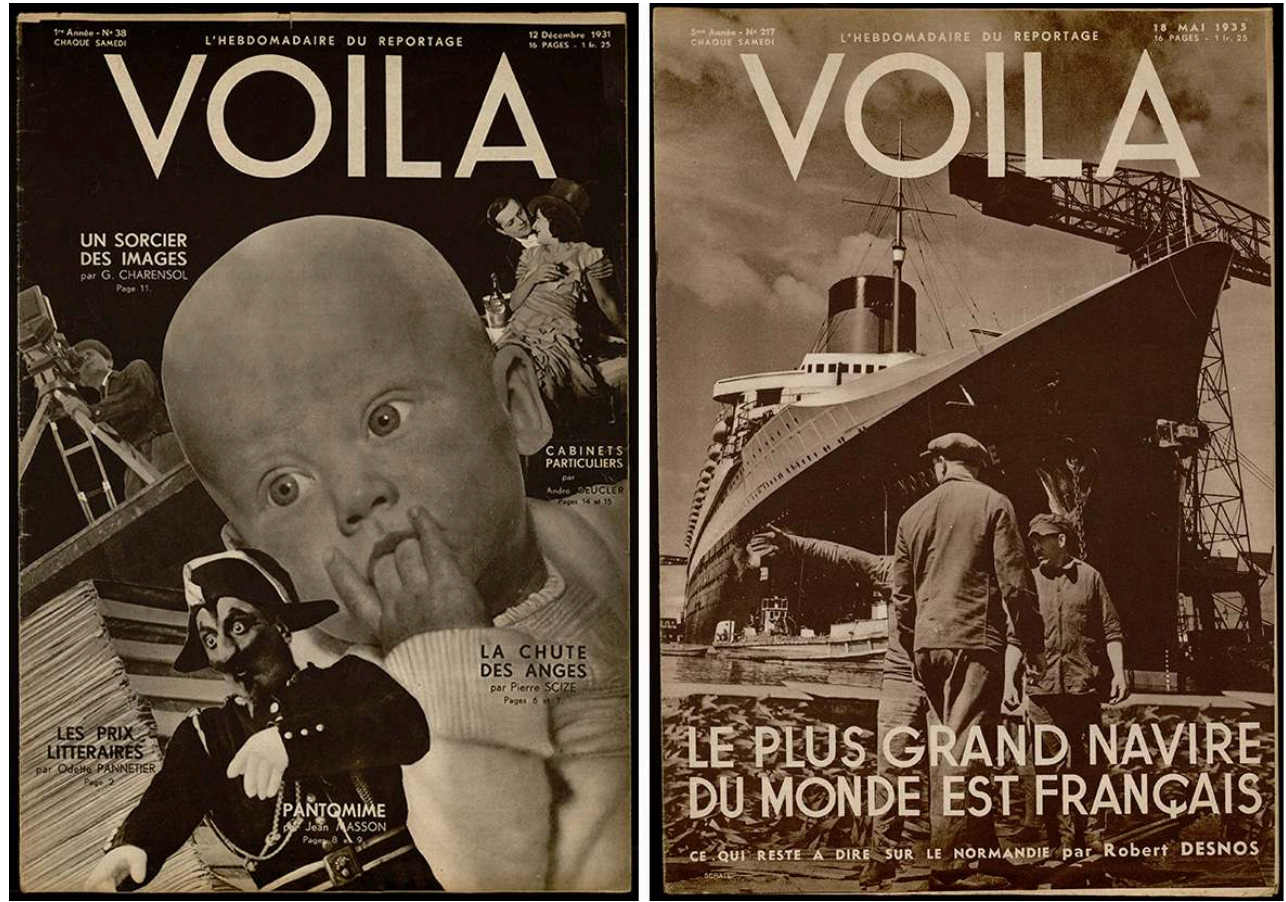

À gauche, couverture du numéro 38 de l'hebdomadaire Voilà, 12 décembre 1931. À droite, couverture du numéro 217 du magazine Voilà, 18 mai 1935

(c) Musée Nicéphore Niépce, ville de Chalon-sur-Saône. 
L'article d'Anne de Mondenard permet de retracer les enjeux qui ont présidé à la présentation de l'exposition «Réalités, un mensuel illustré français (1946-1978) » à la Maison européenne de la photographie (Paris), en 2008, de présenter les choix scénographiques de l'exposition. Créé en 1946, Réalités est un des titres les plus novateurs et les plus influents des années 1950-1960 mais aussi un de ceux qui, pour la période, fut le plus lu. L'exposition ne proposait aucun tirage original, privilégiant la présentation des photographies imprimées et mises en page pour attirer l'attention sur les couvertures, les articles illustrés ou des doubles pages qui installent les photographies dans leur contexte d'usage et de lecture selon une organisation et un dispositif que les commissaires ont voulus attractifs pour le visiteur.

Les deux articles suivants présentent les propositions d'une agence et d'un photographe de presse pour organiser et valoriser leurs fonds photographiques.

Clara Bouveresse présente les enjeux posés par la conservation et la valorisation des archives de l'agence Magnum, coopérative fondée en 1947. Après avoir dressé un état des lieux des collections et de leurs lieux de conservation, elle recense les problématiques soulevées par ces fonds éclatés et met en lumière les politiques de préservation menées par l'agence depuis quelques années. L'article pose une question relative à l'évolution du statut des tirages argentiques à l'aune de la numérisation. Pour l'auteur, la dématérialisation des archives, désormais diffusées en ligne, s'est en effet accompagnée d'une réappréciation plus patrimoniale des tirages. Ces deux mouvements, loin d'être contradictoires, vont de pair pour Magnum qui redéploye les valeurs de l'exclusivité et de la rareté assurant la promotion des photographies de la presse aux cimaises des galeries.

L'article d'Émilie Fromentèze présente la démarche d'un photographe de presse, Patrick Chauvel, soucieux de reconstituer son archive disséminée afin d'en assurer la conservation, la documentation et la valorisation dans le cadre d'une structure qu'il a spécialement créée, aujourd'hui associative mais appelée à évoluer. Cette attitude est révélatrice du désarroi des photographes et particulièrement des photoreporters devant récupérer leurs photographies auprès d'agences et de titres, en assurer la conservation et l'exploitation. L'expérience menée, coûteuse, reste exceptionnelle mais offre des pistes de réflexion.

Cette recherche d'une solution est bien à l'origine de la création du Centre international du photojournalisme à Perpignan, parallèlement au festival «Visa pour l'image ». Frédérique Gaillard revient sur cette structure dont l'ambition est double : valoriser le photojournalisme, accueillir des fonds de photographes inquiets pour le devenir de leur œuvre. Elle rappelle les enjeux de cette conservation qui doit être menée en lien avec l'histoire contemporaine, la sociologie, les sciences de l'information et de la communication, l'anthropologie et l'anthropologie visuelle... À Toulouse, la Ville a travaillé plus de dix ans sur le projet d'acquisition de l'important fonds de Jean Dieuzaide pour aboutir à un accord qui, pour les institutions récipiendaires du fonds, n'est pas sans poser quelques problèmes.

Ce numéro est riche mais il n'épuise pas le sujet. Que tous les contributeurs soient ici remerciés pour la qualité de leurs articles qui permettent de dresser un premier état des lieux des problèmes posés par la gestion de ce type de fonds, qui contribuent par les outils qu'ils proposent à favoriser l'accueil, le traitement et la valorisation de ces ensembles, Quelques contributions manqueront, notamment du côté de producteurs de fonds. Il aurait été intéressant par exemple d'avoir une ou deux contributions de 
responsables de photothèques de journaux, de responsables d'agences de presse. Lors du colloque, ceux-ci étaient présents et sont intervenus. Par ailleurs, le numéro évoque le devenir des fonds argentiques mais le sort des archives photographiques numériques est, en soi, un sujet d'importance qui préoccupe déjà les photographes, les agences et les journaux mais aussi les responsables de collections.

Ce n'est qu'une première étape. La réflexion engagée devra être poursuivie, elle a permis de confronter les méthodes mises en place par les professionnels pour pérenniser et transmettre ce patrimoine essentiel qui contribue à la connaissance de nos sociétés mais aussi à la reconnaissance d'une photographie dont l'usage populaire a souvent réduit l'importance.

De très nombreux grands photographes ont accompagné l'évolution de la presse et ont contribué à son essor et à sa reconnaissance, ils ont aussi écrit dans ses pages leurs plus beaux récits photographiques, publié des photographies devenues iconiques par-delà leur usage premier (fig. 9).

Figure 9

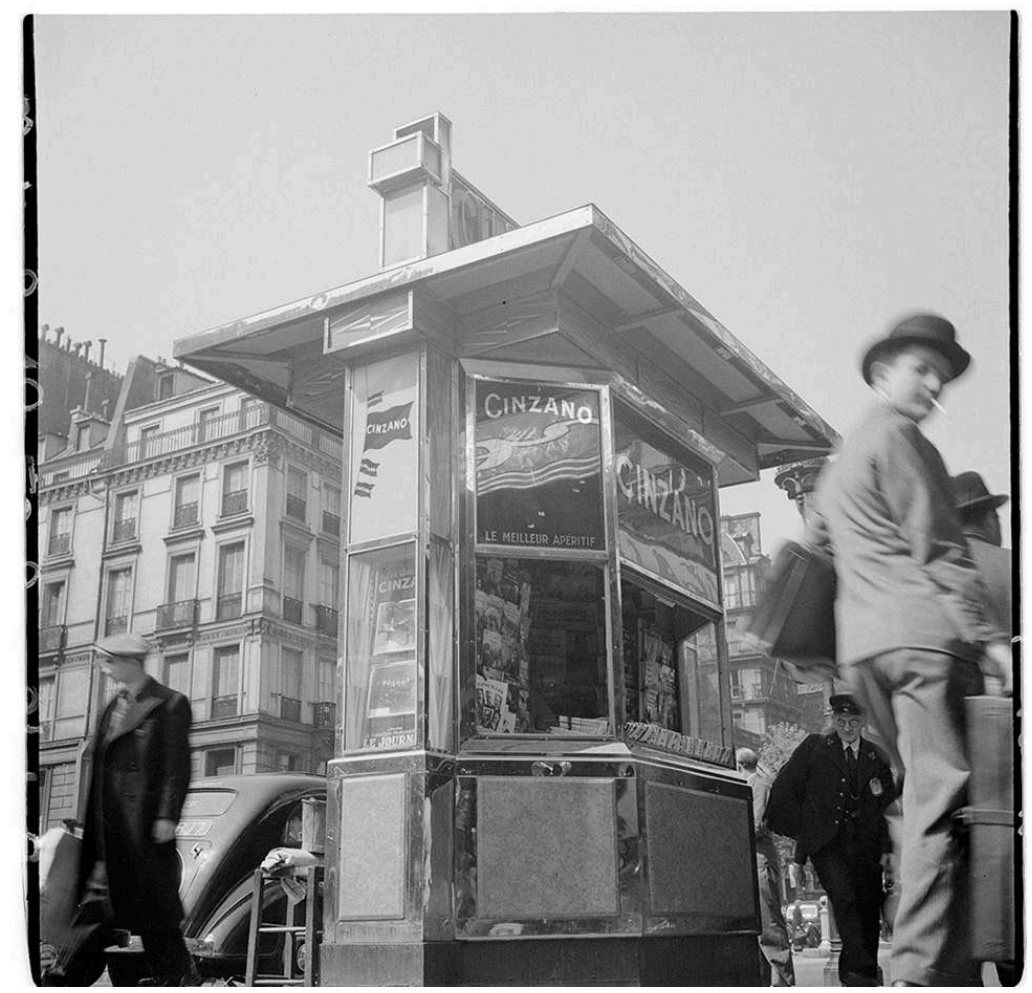

André Papillon, Kiosque à journaux, tirage argentique.

(c) Succession André Papillon, musée Nicéphore Niépce, ville de Chalon-sur-Saône. 


\section{NOTES}

1. - Une première restitution de cette enquête a été faite lors des journées d'études de novembre 2015 sur la base des réponses reçues à un questionnaire envoyé aux archives départementales et municipales entre juin et octobre 2015. Un article rendant plus précisément compte de ses résultats complètera ce numéro.

\section{AUTEUR}

\section{ISABELLE-CÉCILE LE MÉE}

Chargée de mission pour la photographie patrimoniale, direction générale des Patrimoines, ministère de la Culture isabelle.lemee@culture.gouv.fr 